\title{
Tuberculosis in captive Asian elephants (Elephas maximus) in Peninsular Malaysia
}

\begin{abstract}
A cross-sectional study was conducted from 10 January to 9 April 2012, to determine the seroprevalence of tuberculosis (TB) of all captive Asian elephants and their handlers in six locations in Peninsular Malaysia. In addition, trunk-wash samples were examined for tubercle bacillus by culture and polymerase chain reaction (PCR). For 63 elephants and 149 elephant handlers, TB seroprevalence was estimated at $20.4 \%$ and $24.8 \%$, respectively. From 151 trunk-wash samples, 24 acid-fast isolates were obtained, 23 of which were identified by hsp65-based sequencing as non-tuberculous mycobacteria. The Mycobacterium tuberculosisspecific PCR was positive in the trunk-wash samples from three elephants which were also seropositive. Conversely, the trunk wash from seven seropositive elephants were PCR negative. Hence, there was evidence of active and latent TB in the elephants and the high seroprevalence in the elephants and their handlers suggests frequent, close contact, two-way transmission between animals and humans within confined workplaces.
\end{abstract}

Keyword: Captive Asian elephants; Elephants handlers; Mycobacterium tuberculosis; Peninsular Malaysia 